\title{
Long-term survival for COPD patients receiving noninvasive ventilation for acute respiratory failure
}

This article was published in the following Dove Press journal:

International Journal of COPD

24 April 2013

Number of times this article has been viewed

Ingrid L Titlestad'

Annmarie T Lassen ${ }^{2}$

Jørgen Vestbo ${ }^{1,3}$

'Department of Respiratory

Medicine, ${ }^{2}$ Department of Emergency

Medicine, Odense University Hospital,

University of Southern Denmark,

Odense, Denmark; ${ }^{3}$ Respiratory

Research Group, Manchester

Academic Health Sciences Centre,

University Hospital South Manchester

NHS Foundation Trust, University of

Manchester, Manchester, UK
Correspondence: Ingrid Louise Titlestad Department of Respiratory Medicine, Odense University Hospital,

Sdr Boulevard 29, 5000 Odense C,

Denmark

Email ingrid.titlestad@rsyd.dk
Abstract: Implementation of noninvasive ventilation (NIV) as an add-on treatment has been routinely used in a non-intensive care setting since 2004 for patients with chronic obstructive pulmonary disease (COPD) and acute hypercapnic respiratory failure at a university hospital in Denmark. Although randomized controlled trials show lowered mortality rates in highly selected patients with acute exacerbation and respiratory failure, there are only few reports on long-term survival after receiving NIV. We present long-term all-cause mortality data from patients receiving NIV for the first time.

Method: Data from medical records were retrospectively retrieved from all patients receiving NIV for the first time after being admitted acutely to an acute medical ward and further transfer to a respiratory ward with respiratory failure and a diagnosis of COPD in the period January 1, 2005 to December 31, 2007; patients were followed until January 2012. Demographic data collected included age, sex, diagnoses at discharge, and, when present, $\mathrm{FEV}_{1}$; a "not-to-intubate" order was also registered when listed.

Results: In total, 253 patients (143 female, 110 male) received NIV for the first time. The median age was 72 years (range 46-91 years). The 30-day mortality rate was $29.3 \%$. The 5-year survival rate was $23.7 \%$. Women showed a trend towards better survival than men $(25.7 \% \mathrm{vs}$ $19.2 \%, P=0.25)$, and the trend was even more pronounced for patients with COPD.

Conclusion: The mortality rate of patients receiving NIV is high, as expected in a real-life setting, but with a 5-year survival rate of $23.7 \%$ with a trend towards more female than male long-term survivors.

Keywords: COPD, acute respiratory failure, NIV, long-term survival

\section{Introduction}

Randomized controlled studies have shown that noninvasive ventilation (NIV) reduces short-term mortality, risk of intubation and complication rate when given as an additional treatment to selected patients with an acute exacerbation in chronic obstructive pulmonary disease (COPD) complicated by hypercapnic acute respiratory failure. ${ }^{1,2}$ Plant et $\mathrm{al}^{2}$ showed a decrease of the in-hospital mortality rate from $20 \%$ to $10 \%$ when NIV was given to patients with mild acidosis and in a stationary or intermediary ward in a multicenter setting. As with most other treatment modalities, there is likely to be a less noticeable effect when comparing real-life and clinical trial efficacy. This was documented in an audit performed in the UK, ${ }^{3}$ where the aim was to explore factors related to reported high mortality rates.

NIV was implemented as an add-on modality in an acute medical ward and a respiratory ward at Odense University Hospital, Odense, Denmark, in $2004,{ }^{4}$ based on local 
instructions and national recommendations, ${ }^{5}$ and the modality has since been used as a standard additional treatment primarily for patients with hypercapnic acute respiratory failure and verified or presumed COPD. Specifically, patients with arterial measures of $\mathrm{pH}<7.35$ and $\mathrm{PaCO}_{2}>6.0 \mathrm{kPa}$ and mono-organic symptoms of dyspnea, respiratory rate $>25$ and $\mathrm{PaO}_{2}<7.0 \mathrm{kPa}$ without oxygen therapy are eligible for NIV. Exclusion criteria for NIV treatment in the ward are untreated pneumothorax, unstable hemodynamics, and affected alertness, indicating need of intensified monitoring and intubation capability when required.

There are few reports on long-term survival after receiving NIV in a clinical setting, ${ }^{6}$ and the issue of whether there is an advantage by implementing NIV in patients with severe prognosis and expected high mortality rates has not been addressed. The aim of this study was to analyze long-term all-cause mortality following the first years of implementing NIV in a real-life acute medical ward at a university hospital in Denmark, with the null hypothesis that NIV has no impact on long-term survival.

\section{Methods}

All NIV treatments were registered prospectively at the acute medical and respiratory ward of the Odense University Hospital, with patients receiving NIV for the first time in the period January 1, 2005 to December 31, 2007 included for this analysis. Data was retrospectively retrieved from medical records. Baseline data registered were age, sex, when registered in the records, arterial blood gas analyses initiating the indication for NIV ( $\mathrm{pH}$ and $\mathrm{pCO}_{2}$ are presented), diagnoses at discharge, and forced expiratory volume in 1 second $\left(\mathrm{FEV}_{1}\right)$. Lastly, a "not-to-intubate" order was also registered when listed. Patients were included at the time of their first NIV episode, and were followed until death or January 31, 2012 using the Danish Central Person Register to ensure complete follow-up.

\section{Organization of ward}

Patients were admitted to the acute medical ward from either the general practitioner or the emergency ward at the hospital. When patients were suspected of having an acute exacerbation of COPD based on clinical presentation, prior records, arterial blood gas analyses, and chest X-ray, patients were assessed after initial standard treatment with oxygen, inhaled bronchodilator treatment, administration of systemic steroids, and, if needed, antibiotics. Patients either continued standard medical treatment or were provided additional ventilatory support with NIV in the medical acute ward. Criteria for NIV were: (1) arterial blood $\mathrm{pH}<7.35$; (2) $\mathrm{PaO}_{2}<7.0 \mathrm{kPa}$; and (3) $\mathrm{PaCO}_{2}>6.0 \mathrm{kPa}$ in patients with mono-organic symptoms of dyspnea and respiratory rate $>25$. Where possible, patients initiated on NIV were referred to the respiratory ward the next day. Since NIV was initiated in the acute medical ward, standard NIV protocols were followed (initial inspiratory positive airway pressure $10 \mathrm{~cm} \mathrm{H}_{2} 0$ and expiratory positive airway pressure $4 \mathrm{~cm} \mathrm{H}_{2} 0$ ), with oxygen supplement depending on response (aim: peripheral oxygen saturation measure of 90\%-92\%), and changes in pressure were performed depending on the clinical manifestation in accordance with the Danish national guideline. ${ }^{5}$ The protocol on use of NIV remained unchanged in the period of the study. Initiation was done by internal medical staff and a respiratory physician was available for consultation if required.

Patients were monitored with repeated arterial analyses; if the patient's status deteriorated, this patient was discussed with the attending intensive care unit (ICU) doctor, unless the patient did not want ICU treatment or the attending acute medical physician or respiratory physician had issued a "do-not-intubate" order, based on the severity of the underlying illness(es). On a few occasions, NIV was initiated for weaning in patients with prior longer intubation need due to respiratory failure; these patients are reported on separately.

\section{Results}

In the study period 2005-2007, NIV was initiated on 390 occasions: 107 times in 2005, 143 times in 2006, and 140 times in 2007. Of the 302 patients registered, 253 patients received NIV for the first time and were included in this study. Of these, 13 patients received NIV as part of a weaning strategy.

COPD was listed as the primary diagnosis in 216 patients ( $85.4 \%)$; the diagnosis was confirmed by documented spirometry in 182 patients (8\%) and was judged as highly probable in 34 patients $(16 \%)$. Other diagnoses registered at discharge in patients presenting with acute hypercapnic respiratory failure were congestive heart failure, thoracic malignancy, and hypoventilation by other causes (see Table 1). Discharge diagnoses and baseline characteristics are provided in Table 1.

Arterial gas analyses resulting in initiation of NIV was registered in $51.4 \%$ of cases; in these patients, the median $\mathrm{pH}$ was 7.24 (7.07-7.43), and median $\mathrm{pCO}_{2}(\mathrm{kPa})$ was 10.1 (6.0, 20.3).

The 30-day mortality rate for the whole group was $29.3 \%$. After 5 years, $23.7 \%$ of the patients were still alive as shown in Figure 1. For patients with documented COPD, the corresponding 30 -day mortality was $24.3 \%$ and 5-year survival was $23.1 \%$ (see Figure 2). There was no statistically significant 
Table I Demographics on all patients receiving NIV for the first time at a university hospital in Denmark in 2005-2007

\begin{tabular}{|c|c|c|}
\hline & Nonsurvivors & Survivors \\
\hline & Time to death (days) & Observation time (days) \\
\hline & $183(0 ; 13 ; 774 ; 2224)^{*}$ & 2155 (1369; 1782; 244I; 3017)* \\
\hline Patients, $\mathrm{n}$ (male/female) & $198(90 / 108)$ & $55(19 / 36)$ \\
\hline Age (years)* & $74(46 ; 67 ; 80 ; 90)$ & $66(24 ; 58 ; 72.5 ; 91)^{* *}$ \\
\hline COPD confirmed/+probable, $n$ (\%) & $135 / 169(68.2 / 85.4)$ & $47(85.5)$ \\
\hline Documented spirometry, n (\%) & $112(83.0)^{* * *}$ & $32(68.1)^{* * *}$ \\
\hline $\mathrm{FEV}, \%$ of expected (median)* & $27(8 ; 19 ; 34 ; 64)$ & $30(14 ; 21 ; 41 ; 65)$ \\
\hline Ordination of "not-to-intubate," n (\%) & $26(13.1)$ & 0 \\
\hline NIV as weaning after ICU, $\mathrm{n}(\%)$ & $8(4.0)$ & $5(9.0)$ \\
\hline $\mathrm{pH}$ at NIV initiation (median)* & $7.26(7.07 ; 7.21 ; 7.29 ; 7.43)$ & $7.24(7.11 ; 7.18 ; 7.28 ; 7.42)$ \\
\hline $\mathrm{PCO}_{2}$ at NIV initiation, $\mathrm{kPa}$ (median)* & $10.3(6.1 ; 9.0 ; 12.1 ; 18.4)$ & $9.7(6.0 ; 8.8 ; 11.9 ; 20.3)$ \\
\hline \multicolumn{3}{|l|}{ Acute respiratory failure due to } \\
\hline - Congestive heart failure, n (\%) & - $13(6.6)$ & - 0 \\
\hline - Thoracic malignancy, n (\%) & - $3(1.5)$ & $\cdot 0$ \\
\hline - Hypoventilation, n (\%) & - $4 \pi(2.0)$ & - $8 \pi \pi(14.5)$ \\
\hline - Lung fibrosis, n (\%) & - $7(3.5)$ & - 0 \\
\hline - Pneumonia, n (\%) & - $2(1.0)$ & - 0 \\
\hline
\end{tabular}

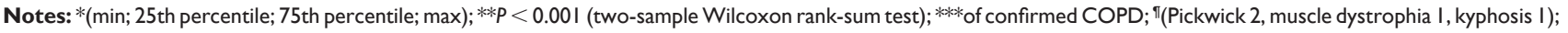
$\pi \pi($ Pickwick 3, kyphosis I, cerebral paresis I, benzodiazepine overdose 3).

Abbreviations: COPD, chronic obstructive pulmonary disease; $\mathrm{FEV}_{1}$, forced expiratory volume in I second; n, number; NIV, noninvasive ventilation.

difference in survival for men and women, although we did see a trend towards better long-term 5 -year survival in females compared to males $(25.7 \%$ vs $19.2 \%, P=0.25$ [logrank]). Presence of COPD did not affect prognosis, but the trend of better long-term 5-year survival in females compared to males was maintained $(27.2 \%$ vs $17.9 \%, P=0.083$ [logrank]).

The only statistically significant differences found between the long-term survivors and patients who died in the observation time were age and issue of a not-to-intubate order. Survivors had a median age of 66 years compared to 74 years in nonsurvivors, and the not-to-intubate order was associated with $100 \%$ mortality. There was no difference in registered $\mathrm{pH}$ or $\mathrm{pCO}_{2}$ between survivors and nonsurvivors at initiation of NIV.

Descriptive data of COPD patients versus non-COPD patients receiving NIV for the first time are shown in Table 2, and supplementary information in Figure 1.

\section{Discussion}

Data on long-term survival of patients receiving NIV for the first time revealed an expected high 30-day mortality rate, considerably higher than that seen in pivotal trials of NIV

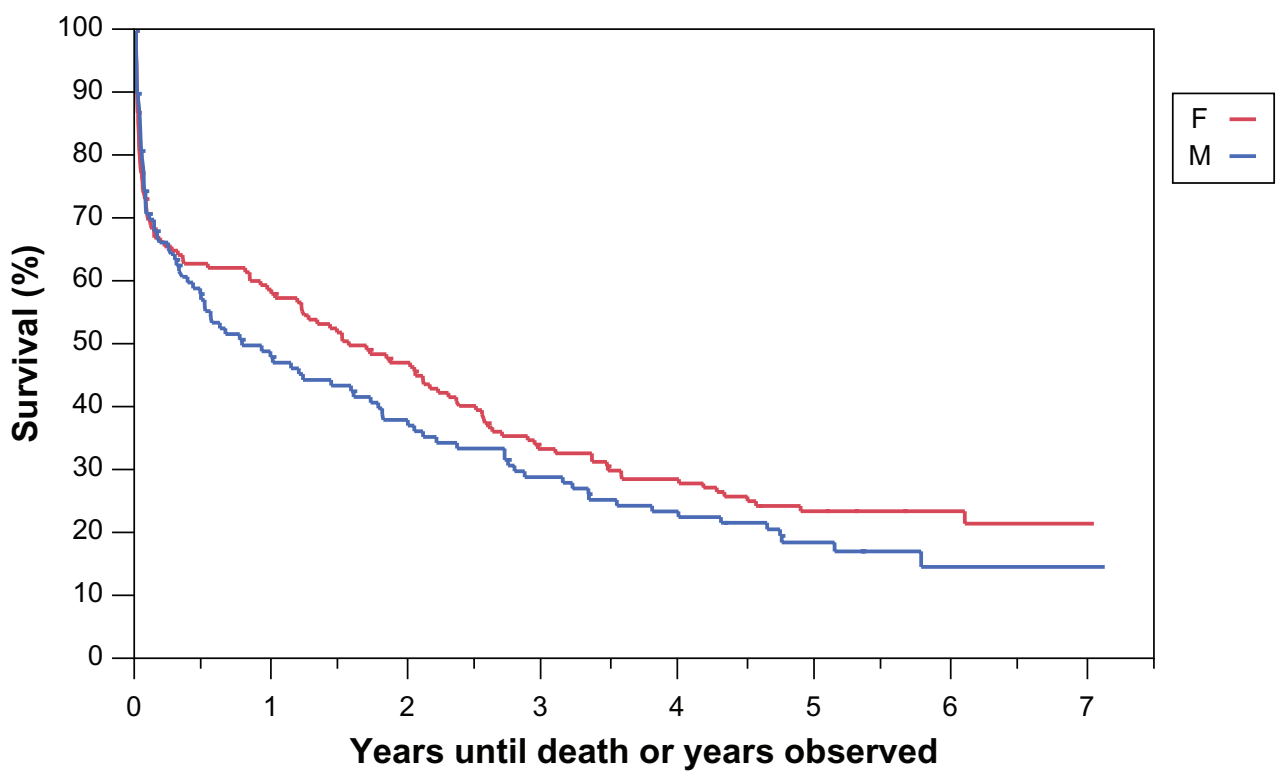

Figure I Survival plot of patients admitted to a respiratory ward (2005-2007) receiving noninvasive ventilation for the first time. Notes: Total 253 patients ( 143 female and 110 male). 5 -year survival for females $25.7 \%$ and males $19.2 \%$. $P=0.25$ (logrank). 


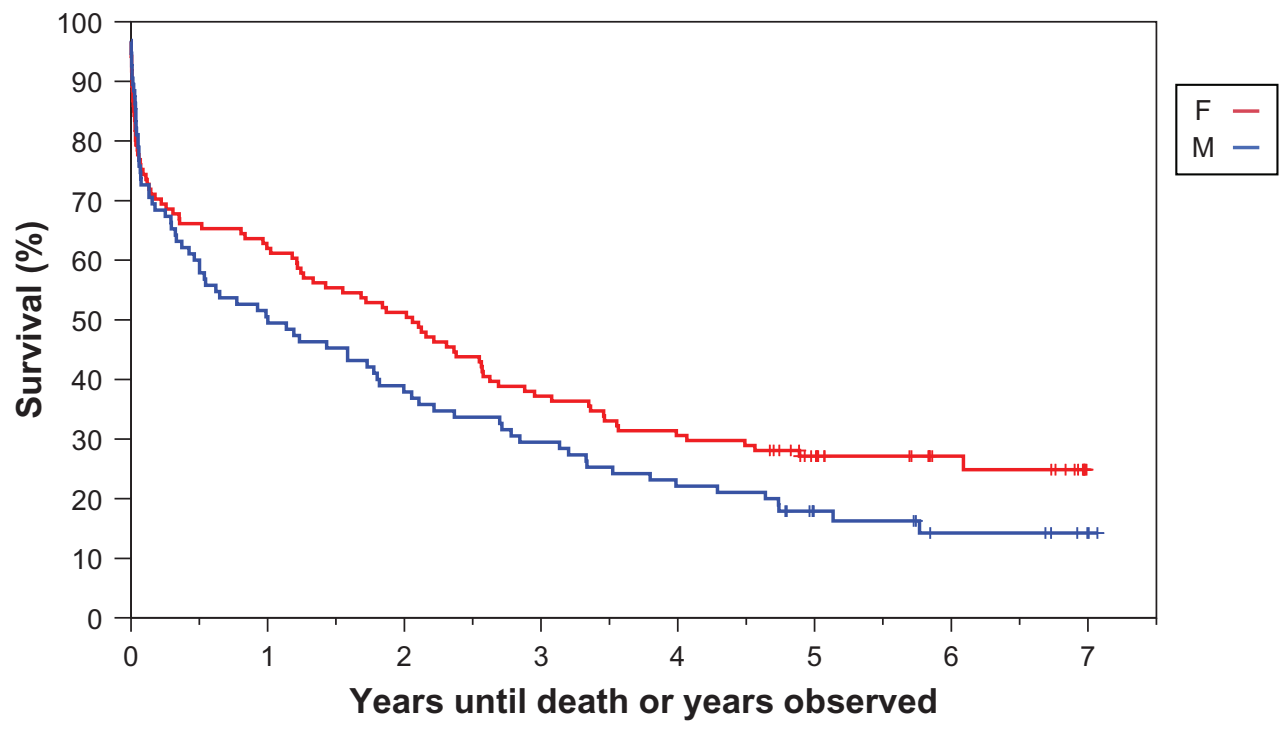

Figure 2 Survival plot and observation time (years) of COPD patients admitted to a respiratory ward (2005-2007) receiving noninvasive ventilation for the first time. Notes: Total 216 patients (143 female and I 10 male). 5 -year survival for females $27.9 \%$ and males $17.9 \%$. $P=0.083$ (logrank).

in $\mathrm{COPD},{ }^{1,2}$ but in line with other observational audits and studies. ${ }^{3,6,7}$ However, the 5-year survival rate was higher than earlier anticipated, which could be due to more stringent selection criteria for initiation of NIV, and the fact that the study was only conducted in one center, giving patients more homogenous treatment. One significant difference between survivors and nonsurvivors was age, survivors being, as a group, younger than the group of patients who died, but the oldest patient (91 years) registered in this study was alive at the end of 5 years of observation. We observed a trend of better long-term survival in females compared to males, in the whole NIV group and especially in patients with COPD, but further studies are needed to evaluate confounding variables. Unfortunately, the registration of comorbidities was not done routinely or stringently, but, in the last couple of years, more focus has been made on screening and registration of comorbidities in COPD, a benefit for future surveys. Historically, males have had a higher exposure of cigarette smoke and

Table 2 Survival and observation time of COPD patients versus non-COPD patients receiving NIV for the first time

\begin{tabular}{|c|c|c|}
\hline & COPD & Non-COPD \\
\hline Patients, $\mathrm{n}$ (male/female) & $216(95 / I 2 I)$ & $37(14 / 23)$ \\
\hline Age, years (median)* & $72(45 ; 66 ; 79 ; 91)$ & 7I $(24 ; 62 ; 80 ; 88)$ \\
\hline $\begin{array}{l}\text { Mean survival of mortal } \\
\text { patients, days (median)* }\end{array}$ & $227(0 ; 15 ; 810 ; 2224)$ & $15(0 ; 4 ; 177 ; 1302)$ \\
\hline $\begin{array}{l}\text { Mean observation time } \\
\text { of survivors, days (median)* }\end{array}$ & $\begin{array}{l}2097 \text { (I707; I822; } \\
2509 ; 2582)\end{array}$ & $\begin{array}{l}2002(1609 ; 1728 ; \\
24 \mid 2 ; 2508)\end{array}$ \\
\hline
\end{tabular}

Note: *(min; 25 th percentile; 75th percentile; max).

Abbreviations: COPD, chronic obstructive pulmonary disease; NIV, noninvasive ventilation. occupational lung irritants ${ }^{8}$ and these factors could be contributing to the trend of worse long-term survival.

It is well known that patients entered into clinical trials often differ significantly from patients in the usual clinical setting. In contrast to randomized controlled studies, this patient-based study included a much less selected group of patients having more comorbidities presenting with acute hypercapnic respiratory failure. Patients were not limited to patients with respiratory failure due to exacerbation in COPD, and, a priori, one could assume that this would affect prognosis. Patients with other diagnoses included patients with clearly reversible disorders, such as overdoses of benzodiazepines, but several survivors were patients with other less morbid diagnoses than COPD. Despite the disadvantages of retrospective and therefore less complete data retrieval from records (especially concerning arterial blood analyses), survivors compared to nonsurvivors were similar with regards to $\mathrm{COPD}$ and, when registered, $\mathrm{FEV}_{1}, \mathrm{pH}$, and $\mathrm{pCO}_{2}$. In the non-COPD patients especially severe diseases such as congestive heart failure, thoracic malignancy, and end-stage fibrosis, were present among nonsurvivors but not at all present in survivors. In several of these cases, NIV was given as a last treatment option in patients with a not-to-intubate order. It seems apparent that patients labeled "not-to-intubate" have a particularly poor prognosis and, based, on our findings probably did not benefit from the treatment.

The study included all patients initiated in an acute medical ward setting or a respiratory ward. Unlike two previous prospective studies by Berkius et al, ${ }^{6}$ including 93 COPD patients in a multicenter ICU setting, and Harris et al, ${ }^{7}$ report- 
ing use of noninvasive ventilation (continuous positive airway pressure and bilevel positive airway pressure) in clinical practice over 5 years, we have not included patients receiving the modality of NIV initiated only in the ICU setting. Berkius et $\mathrm{al}^{6}$ reported survival data (5-year) for COPD patients receiving NIV in the ICU (17\%), which was lower for patients needing intubation (6\%). A national British COPD audit from 2008 on acidosis, NIV, and mortality in hospitalized patients with COPD exacerbations, including 232 hospital units and 9716 patients, showed that $20 \%$ of the patients had acidotic artery blood analyses at admission. ${ }^{3}$ NIV treatment in clinical practice was given to severely ill patients, many with mixed metabolic acidosis. Further, patients fulfilling clinical criteria for NIV treatment failed to receive NIV, and others received it inappropriately. NIV was also seen used as a ceiling of treatment in which efficacy was uncertain.

The primary focus of this present study has not been evaluation of the indication for NIV treatment; since 2008, an annual national surveillance of patients with COPD (Dansk Register for KOL [DrKOL]) has been launched, registration practice (International Classification of Diseases [ICD]-10) has been reviewed, and audits performed. In Denmark, treatment with NIV was provided to $9 \%$ of all COPD patients admitted with an exacerbation in 2009 and $10 \%$ in 2010 and 2011.9

\section{Conclusion}

Our study confirms that patients with hypercapnic respiratory failure are a high-risk group, often with a poor prognosis. It casts some doubt as to what is achievable from NIV in a non-ICU setting and suggests that regular audits are required to ensure that the right patients are offered the right treatment when admitted with mild hypercapnic respiratory failure. However, the 5-year survival rate was higher than earlier anticipated, justifying the broad approach to NIV treatment in this setting. The high 30-day mortality rate for all NIVinitiated patients was higher (29.3\%) than for the group of patients with COPD (24.3\%), and this supports the notion that NIV should be dedicated primarily to patients with COPD in exacerbation. The 30-day mortality rate for COPD patients receiving NIV was similar in an audit from 2010, and patients with $\mathrm{pH}<7.25$ had a worse outcome than patients presenting with higher $\mathrm{pH}$ at NIV initiation (unpublished data). Monitoring of patient outcomes in a real-life setting is necessary to ensure optimal selection of patients for NIV in a non-ICU setting.

\section{Disclosure}

The authors report no conflicts of interest in this work.

\section{References}

1. Celikel T, Sungur M, Ceyhan B, Karakurt S. Comparison of noninvasive positive pressure ventilation with standard medical therapy in hypercapnic acute respiratory failure. Chest. 1998;114;1636-1642.

2. Plant PK, Owen JI, Elliott MW. Early use of non-invasive ventilation for acute exacerbations of chronic obstructive pulmonary disease on general respiratory wards: a multicentre randomised controlled trial. Lancet. 2000;355:1931-1935

3. Roberts CM, Stone RA, Buckingham RJ, Pursey NA, Lowe D; the National Chronic Obstructive Pulmonary Disease Resources and Outcomes Project implementation group. Acidosis, non-invasive ventilation and mortality in hospitalised COPD exacerbations. Thorax. 2011;66:43-48.

4. Titlestad IL, Madsen HD. Implementation of non invasive ventilation in respiratory ward in patients with exacerbations of chronic obstructive pulmonary disease. Ugeskr Laeger. 2008;21;170(4):240-243. Danish.

5. Hansen EF, Fabricius P, Titlestad IL, Wessels J. Chronic Obstructive Pulmonary Disease (COPD) in Exacerbation and Noninvasive Ventilation (NIV) Treatment. Danish Lung Diseases Society; 2008. Available from: http://www.lungemedicin.dk/retningslinier/Godkendte\%20retningslinier/ KOL\%20i\%20exacerbation\%20og\%20NIV.pdf. Accessed July 3, 2012. Danish.

6. Berkius J, Sundh J, Nilholm L, Fredrikson M, Walther M. Long-term survival according to ventilation mode in acute respiratory failure secondary to chronic obstructive pulmonary disease: a multicenter, inception cohort study. J Crit Care. 2010;25:539.e13-539.e18.

7. Harris C, Saskin R, Burns KE. Noninvasive ventilation initiation in clinical practice: a six-year prospective, observational study. Can Respir J. 2010;17(3):123-131.

8. Trupin L, Earnest G, San Pedro M, et al. The occupational burden of chronic obstructive pulmonary disease. Eur Respir J. 2003;22:462-469.

9. DrKOL: Dansk register for Kronisk Obstruktiv Lungesygdom. National Arsrapport 2011. January 1, 2011-December 31, 2011 [Danish Register of Chronic Obstructive Pulmonary Disease. National Annual Report 2011. January 1, 2011-December 31, 2011]. Version 3. Århus, Denmark: Det Nationale Indikatorprojekt; 2012. Available from: https:// www.sundhed.dk/content/cms/90/4690_drkol_\%C3\%A5rsrapport2011_ kommenteret_v3_20120426.pdf. Accessed July 3, 2012. Danish.
International Journal of COPD

\section{Publish your work in this journal}

The International Journal of COPD is an international, peer-reviewed journal of therapeutics and pharmacology focusing on concise rapid reporting of clinical studies and reviews in COPD. Special focus is given to the pathophysiological processes underlying the disease, intervention programs, patient focused education, and self management protocols.

\section{Dovepress}

This journal is indexed on PubMed Central, MedLine and CAS. The manuscript management system is completely online and includes a very quick and fair peer-review system, which is all easy to use. Visit http://www.dovepress.com/testimonials.php to read real quotes from published authors. 